\title{
Surgical Management of Proximal Humerus Fractures - Assessment of Results
}

\author{
Dr.B.Venkat Reddy, M.S(Ortho) ${ }^{1}$, Dr. Saketh Kolla, M.S (Ortho $)^{2}$ \\ ${ }^{I}$ (Kamineni Institute Of Medical Sciences, Narketpally)
}

\begin{abstract}
:
Background: Proximal humeral fracture whether caused by trauma or related to osteoporosis, requires carefully planned individual treatment. The choice of technique and devices depends on quality of bone, soft tissue, age and reliability of patients. However the goal of proximal humerus fracture fixation should be stable reduction, allowing early motion of fracture.

Objectives: To analyze fractures of the proximal humerus that were treated either with the proximal humerus internal locking system (PHILOS) locking plate or percutaneous $K$ wire and document their clinical and functional outcome.

Methods: The study includes patients with proximal humerus fractures who underwent Open reduction and internal fixation with PHILOS locking plate or Percutaneous K-wire fixation.

Functional outcome was evaluated by Constant-Murley score. Patients were followed up at 6 weekly interval until fracture union and at once at 1 yr after the surgery

30 cases were studied, cases were selected randomly for both PHILOS and percutaneous $k$ wire fixation, each 15 cases.

Results: $33.34 \%$ patients treated with open reduction and internal fixation with PHILOS plating had good to excellent results, $46.6 \%$ of patients had fair results and the remaining $20 \%$ had poor results.

$40 \%$ of patients treated with closed reduction and $K$ wire fixation had good to excellent results, $40 \%$ of patients had fair results and remaining $20 \%$ had poor results.

Conclusion: The results of surgical treatment of proximal humerus fractures in both the groups (percutaneous pinning and PHILOS plating) are satisfactory with good functional outcome.
\end{abstract}

Keywords: Proximal humerus, Constant-Murley score, PHILOS , $K$ wire

\section{Introduction}

Proximal humeral fractures are the second most common upper-extremity fracture and the third most common fracture after hip fractures and distal radial fractures, in patients who are older than sixty-five years of age. They account for about $5 \%$ of all injuries to appendicular skeleton 1 . Although the overwhelming majority of proximal humeral fractures are either non-displaced or minimally displaced and can be treated with sling immobilization and physical therapy ${ }^{2}$, approximately $20 \%$ of displaced proximal humeral fractures may benefit from operative treatment ${ }^{3}$. Many surgical techniques have been described, but no single approach is considered to be the standard of care. ${ }^{4}$

Over the last 3 decades, various modalities of fixations have evolved for the proximal humerus fractures (transosseous suturing, percutaneous pinning, tension band wiring, plating, rush nailing, arthroplasty). The proximal humerus locking system plate has been developed to improve screw fixation in osteoporotic bone and to minimize soft tissue dissection. The type of fixation used depends on the patient's age, activity and bone quality, the fracture type and the surgeons technical ability. If the fracture reduction is achieved by manipulation but cannot be maintained, percutaneous K- wire fixation is performed. Recent advances in fracture fixation technologies have led to the development of fixed-angle locked plates that maintain angular stability under load.

The treatment is more controversial for articular fractures which carry a high risk of the humeral head necrosis. In Neer's clasification, these are two part, three-part and four-part fracture and those with dislocation of head of humerus. A review of published result suggests that there is no universally accepted form of treatment. Conservative management may be associated with non-union, malunion and avascular necrosis resulting in painful dysfunction.

Proximal humeral fracture whether caused by trauma or related to osteoporosis, requires carefully planned individual treatment. The choice of technique and devices depends on quality of bone, soft tissue, age and reliability of patients. However the goal of proximal humerus fracture fixation should be stable reduction, allowing early motion of fracture.

This study is conducted to analyze fractures of the proximal humerus that were treated either with the proximal humerus internal locking system (PHILOS) locking plate or percutaneous K wire and documents their clinical and functional outcome. 


\section{Ii. Materials And Methods}

The study purpose to include patients with proximal humerus fractures admitted and examined according to protocol, associated injuries noted. Clinical and radiological evaluation done. Fractures classified using Neer's classification. Routine investigations carried out to get fitness for surgery. Patients underwent Open reduction and internal fixation with PHILOS locking plate or Percutaneous K-wire fixation under general anaesthesia/ brachial block. Post operative physiotherapy followed according to protocol, to evaluate the functional outcome. Patients will be followed up at 6 weekly interval until fracture union and at once at $1 \mathrm{yr}$ after the surgery 30 cases were studied, cases were selected randomly for both PHILOS and percutaneous k wire fixation, each 15 cases.

\section{Inclusion Criteria}

1. Patients of age above $18 \mathrm{yrs}$.

2. Two part, three part and four part fracture of proximal humerus

\section{Exclusion Criteria}

1. Children and adolescent patients less than $18 \mathrm{yrs}$

2. Pathological fractures

3. Patients with compound fractures

On admission of the patient a careful history was elicited from the patients and/or attendants of injury and the severity of trauma. The patients were then assessed clinically to evaluate their general condition and the local injury. The general condition of the patient and the vital signs were recorded. The local examination of injured shoulder was done for swelling, deformity, loss of function and altered attitude. Any nerve injury was also looked for and noted. Axillary nerve assesed by looking for anaesthetic patch over lateral aspect of shoulder.

Radiograph of proximal humerus i.e., antero-posterior view and axillary view taken and fractures were classified according to Neer's classification. Next the limb was immobilized in U slab and arm-pouch. The patient was taken for surgery after routine investigation and after obtaining physician fitness towards surgery. The investigations are as follows. Hb\%, RBS, blood urea, serum creatinine, HIV, HBsAg and ECG.

The consent for surgery was also taken from the patient and attendants after explaining the procedure and possible complications.

\section{Postoperative management:}

- All patients are immobilized in arm pouch with cuff and collar sling.

- Appropriate antibiotics and analgesics were used.

- Immediate post operative radiographs were taken to determine the bone alignment and maintainance of reduction.

- Passive range of motion and pendulum exercises are begun immediately depending on pain from third week.

- K-wires are removed at about 6-8 weeks.

\section{Follow up:}

All patents were followed every week in first month and every 2-3 weeks for 6months.

- The active range of motion were started at 1-2 weeks. postoperatively, depending on stability of osteosynthesis and bone quality.

- The sling is discontinued by 8-12 weeks depending upon fracture stability.

- Further follow ups were at 8 weeks and 12 weeks and 24 weeks.

- The patients were examined clinically and radiologically. Assessed for range of motion and bony union and complication.

- The patient with shoulder stiffness given physiotherapy for 1 week to 15 days on outpatient basis.

\section{Functional results:}

The final result were evaluated using Constant-Murley score.

This system base on 100 point score composed of a number of individual parameters. The subjective parameters assess the degree of pain the patient experiences and the ability to perform normal tasks of daily living as they apply to him or her in both activity and position related terms. Both of these assessments are subjective and are carried out independently prior to objective testing of active motion range and shoulder power. 


\section{Results}

In our study most of the patients $40 \%$ belong to the age group of $>50 \mathrm{yrs}$. $30 \%$ patients belong to the age group 41-50. The average age of the patient was 47.93 years. Sex distribution was comparable between males and females corresponding to $53.33 \%$ and $46.6 \%$ respectively. Male: Female sex ratio is 1.14:1. Most of the patients were affected on the right humerus corresponding to $53.33 \%$ and on left side in $46.6 \%$ patients. $56.66 \%$ of our patients had suffered a domestic fall and $43.3 \%$ were involved in motor vehicle accidents.

Fall resulted in three part fracture in 6 patients; Road Traffic Accident resulted in two part fracture in 7 cases. The association of type of fracture and mode of injury is insignificant. In our study, in the age group $>50 \mathrm{yrs}$ the commonest mode of injury is by fall (domestic). In the age group 19-30yrs the commonest mode of injury is by RTA. There is significant association of mode of injury and age. $p<0.05$ which shows significant association.

In our study we had $46.66 \%$ of patients with 2 part fracture and $36.66 \%$ of patients had 3 part fractures. $16.66 \%$ of patients had 4 part fracture. $71.4 \%$ patients with two part fractures were treated using closed reduction and k- wire fixation. $63 \%$ of patients with three part fractures are treated with open reduction and internal fixation with PHILOS plating. 80\% of patients with four part fractures are treated with open reduction and internal fixation with PHILOS plating. The patients treated with open reduction and internal fixation with PHILOS plating, Impingement of the implant with restriction of movements was present in 2 cases and Varus malunion is seen in 1 patient.

In patients treated with percutaneous pinning the complications noted are pin tract infection in 3 of patient and varus malunion in 2 patients. The mean constant Murley score for patients treated with open reduction and internal fixation with PHILOS plating is $68.13 \%$ compared to Percutaneous pinning which is 71.9\%. $\mathrm{p}>0.05$, which refers that comparision between two groups is insignificant. In the present study $33.34 \%$ patients treated with open reduction and internal fixation with PHILOS plating had good to excellent results, $46.6 \%$ of patients had fair results and the remaining $20 \%$ had poor results. $40 \%$ of patients treated with closed reduction and $\mathrm{K}$ wire fixation had good to excellent results, $40 \%$ of patients had fair results and remaining $20 \%$ had poor results.

\section{Discussion}

The operative treatment of proximal humeral fractures is a therapeutic challenge for orthopaedic surgeons. Most of the proximal humerus fracture which are un-displaced can be treated conservatively. Even if the injury is thoroughly analyzed and the literature is understood, treatment of displaced fracture or fracture dislocation is difficult. The result is related to restoration of anatomical alignment, and if the fracture is treated conservatively, a functional deficit will certainly develop and may be associated with pain. The external support is difficult to apply effectively because fracture site is adjacent to trunk.

Many studies have shown that the displaced fracture of the proximal humerus have a poor functional prognosis when left untreated because of severe displacement of fragments. ${ }^{1-5}$ Numerous investigators have described the various surgical treatments for displaced proximal humerus fracture. There is no consensus on optimal treatment of displaced proximal humerus fractures which account for about $20 \%$ of fractures. In some studies, the objective functional results of conservative treatment have been unsatisfactory. The fractures are defined by variety of classification systems. The difficulty in accurately classifying the fracture creates problems in reporting outcome and also none of the system gives clear prognosis and direction of treatment.

Age Incidence: The average age incidence in present series of 30 patients analyzed, was 47.93 years, which was consistent with the age incidence in studies done by Kenneth A. Egol et al, ${ }^{29}$ (61 years) and the average age incidence in C. Gerber et al, ${ }^{23}$ study was 44.9 years. In present series $12(40 \%)$ out of 30 Patients were age group of > 50 years and $9(30 \%)$ patients in $41-50$ age group. Majority of the patient in our group are elderly in our study

In present study the association between age and mode of injury shows that the common mode of injury in the age group 50yrs is by fall (domestic). The common mode of injury in the age group between 19-30yrs is road traffic accidents.

\section{SEX Incidence}

Further as compared with other studies, present study showed a higher incidence of fractures in men than in women. The gender ration was 1.14:1.this higher ration can be explained by a higher involvement of male in day to day activities in compare to female.

\section{Mode of injury:}

Motor vehicle accidents constitute a major cause of musculosketetal trauma worldwide. In our country too, it happens to be very common and is reflected in present study, the second most common cause after the domestic fall. 
Rose SH et al, in their study of proximal humerus fracture, have reported $80 \%$ of cases the mode of injury was minor fall in a patients aged above 40 years and especially in osteoporotic females ${ }^{4}$. Herbert Resch et al in their study 27 patients with three part and four part fracture,24 patients had history of high energy trauma. ${ }^{32}$ Fall resulted in three part fractures in 6 cases, road traffic accident resulted in two part fractures in 6 cases and three part fracture in 5 cases and four part fractures in 2 cases.

\section{Side afeected:}

In our present study fracture occurred on right side in 16 patients and on left side in 14 patients .C. Gerber reported ,in their series of 34 fractures 16 were on left side and 18 were on right side. ${ }^{23}$

\section{Period between injury and time of surgery:}

The average interval between fracture and surgery was 3.63 days in our study.Average interval between fracture and surgery was 3.2 days in Gerber C.et al Study. ${ }^{23}$

\section{Type Of Fracture}

The study of type of fracture in present series revealed, 14 (46.66\%) were two part fractures, 11 (36.66\%) were three part fractures, $05(16.66 \%)$ was four part fracture. In studies done by Rizwan Shahid et $\mathrm{al}^{28}{ }^{28}$ in a series of 50 patients studied $11(22 \%)$ were two part fractures, $21(42 \%)$ were three part fractures and $18(36 \%)$ were four part fractures. In another study by MA Fazal et al, ${ }^{32}$ of 27 cases $13(48 \%)$ were two part fractures, $12(44.5 \%)$ were three part fractures and $2(7.5 \%)$ were four part fractures indicating that the incidence of type of fracture is nearly consistent with the studies in literature.

\section{Complications :}

Secondary displacement and malunion occurred in three cases at surgical neck. It usually involves to anterior angulation and varus deformity, decreasing neck shaft angle $<120^{\circ}$. It was probably due to communition of underlying osteoporotic bone which may go impaction at the fracture site after reduction leading to varus malunion.

Two patients in PHILOs group had plate impingement and limitation of abduction, its hardware related complication, improper plate positioning may have lead to impingement. Three patients had pin track infection in percutaneous K- Wire fixation series which are treated with appropriate antibiotics and it didn't result in any loosening of k-wire. Pin track infection is the commonly encountered complication in percutaneous pinning.

- Both complications in PHILOS group i.e. varus malunion and impingement lead to poor results.

- In percutaneous group pin track infection is commonest complication where $66 \%$ of patients had fair to excellent result and the remaining $33 \%$ had poor results.

V. Tables: comparision of results in studies Treatedwith Philos Plating

\begin{tabular}{|c|c|}
\hline Study & $\begin{array}{c}\text { Constant Murley } \\
\text { Score }\end{array}$ \\
\hline Gerber.,Et Al. $^{9}$ & 78 \\
\hline Monnot Et Al. $^{19}$ & 66.5 \\
\hline Felix Brunner Et Al. $^{38}$ & 72 \\
\hline Present Study $^{38}$ & 68.13 \\
\hline
\end{tabular}

Comparision Of Results In Studies Treated Percutaneous K-Wire Fixation

\begin{tabular}{|c|c|}
\hline Study & Constant Murley Scores \\
\hline Jay D. Keener Md Et Al. $^{52}$ & 73.9 \\
\hline Bruner.Aetal. $^{53}$ & 73.6 \\
\hline Present Study $^{2}$ & 71.9 \\
\hline
\end{tabular}

\section{Conclusion}

In both the groups of percutaneous pinning and plate fixation, comparatively minor differences were detected with regard to functional outcome. The results of surgical treatment of proximal humerus fractures in both the groups (percutaneous pinning and PHILOS plating) are satisfactory with good functional outcome. The most common complication in open reduction and plate fixation is plate impingement, leading to limitation of abduction. Closed reduction and percutaneous pinning have advantage in retaining the vascularity of the humeral head.

- It can be used for Un-displaced two, three or four part fracture of the proximal humerus without communition, in the younger age groups.

- Elderly who are unfit for major surgery.

Complication faced are pin track infections and loss of reduction. 
Conflict of interest: The authors declare that they haven't any conflict of interest.

\section{References}

[1]. Court Brown CM,Caesar B. Epidemiology of adult fractures: A review Injury 2006;37:691-697.

[2]. Terry Canale's Campbell's Operative Orthopaedics, Vol-3: 9th edition, 1998 Mosby Publishers, USA Pg $2286-2296$.

[3]. Steven .H.Rose Joseph Melton Bernard.F.Morrey et al Epidemiological features of humeral fractures Clin. Orthop-1982:168:24-30.

[4]. RL Sahu : Philos Locking plates in proximal humerus fractures literature review..The Internet Journal of Health. 2010 Volume 11

[5]. Bigliani LU, Flatow EL, Pollock RG. Fractures of the proximal humerus In: Rockwood CA, Green DP, Bucholz RW, Heckman JD, eds. Fractures In adults. Philadelphia, etc: Lippincott-Raven, 1996: 1055-107.

[6]. Buchanan JJ. IV. Fracture through the anatomical neck of the humerus with dislocation of the head. Ann Surg 1908; 47:659-71.

[7]. Lous U., Bigiliani, Chapter 9 The shoulder, Vol-1 ed. Charles Rockwood, Frederick A. Fractures of proximal humerus. In Rockwood CA, Matsen. Philadelphia: W.B. Saunders, 1990: p278-334.

[8]. Neer CS. Displaced proximal humeral fracture: Part 1: Classification and evaluation. J Bone Joint Surg (Am) 1970; 52-A: $1077-89$.

[9]. Neer CS. "Displaced proximal humeral fractures. Par t II. Treatment of three part and four part displacement". J Bone Joint Surg , 1970; 52A : 1090-11.

[10]. Paavolainen P, Bjorkenheim JM, Slatis P, and Paukku P. "Operative treatment of severe proximal humeral fractures". Acta Orthop Scand, 1983; 54: 374-379.

[11]. Siebler G, Kuner EH , "Late results following the surgical treatment of proximal humerus fractures in adults". Unfall chirurgie 1985; 11(3): 119-127.

[12]. Kristiansen B and Christensen SW : Plate fixation of proximal humeral fractures, Acta Orthop Scand, 1986; $57: 320-323$.

[13]. Mourdian WH., "Displaced proximal humeral fractures. Seven years experience with a modified zickel supracondyalr device". Clin Orthop 1986; 212: 209-218.

[14]. Moda SK, Chadha NS, Sangwan SS, Khurana DK, Dahiya AS, SiwachRC. "Open reduction and fixation of proximal humeral fr actures and fracture dislocations". J Bone Joint Surg 1990; 72-B: 1050-1 052.

[15]. Szyszkowitz R, Seggl W, Schleifer P, Cundy PJ. Proximal humeral fractures : management techniques and expected results. Clin Orthop, 1993; 293: 13-25.

[16]. Robinson CM, Christie T. Two part proximal humeral fracture: A review of operative treatment using two techniques. Injury, 1993; 24(2): 123-125.

[17]. Zyto K, Wallace WA, Frostick SP, Preston BJ. Outcome after hemiarthroplasty for three and four part fracture of the proximal humerus. J Shoulder Elbow Surg, 1998; 7: 85-9.

[18]. Koval KJ, Blair B, Takie R, Kummer FT, Zuckerman D. "Surgical neck fractures of proximal humerus; a laboratory evaluation of ten frixation techniques". J Trauma 1996; 40(S): 778-783.

[19]. Hessmann M, Gehling H, Gotzenl, "Plate fixation of proximal humerus fracture with indirect reduction; surgical technique and results using the shoulder score.". Injury 1999; 30: 453-462.

[20]. Hintermann B, Trouillier HH, Schufer D. "Rigid internal fixation of fractures of the proximal humerus in older patients". J Bone Joint Surg (Br), 2000 Nov; 82(8): 1107-1112.

[21]. Wijgman A.J, Roolker W,Patt T W et al, open reduction and internal fixation of three and four part fractures of proximal humerus. Scientific Article November 01, 2002.

[22]. Jan-Magnus Björkenheim, Jarkko Pajarinen et al, "Internal fixation of proximal humeral fractures with a locking compression plate" Acta Orthop Scand 2004; 75 (6): 741-745.

[23]. Gerber C, Worner CM, Vienne P. "Interal fixation of complex fractures of the proximal humerus". J Bone Joint Surg (Br), 2004 Aug; 86(60:P 848- 855.

[24]. C.P.Charalambous ,I.siddiqe, et al"Proximal humerus internal locking for the treatment of proximal humerus fracture" Archieve of orthopaedic and trauma surgery(2007)127:205-210.

[25]. Reto Babst,Flexi Brunner" Plating in proximal humerus fractures" European Journal of trauma and emergency surgery 2007:33,345-56.

[26]. Moonot P, Ashwood N, Hamlet M., Early results for treatment of three- and four- part fractures of the proximal humerus using the PHILOS plate system , J Bone Joint Surg Br. 2007 Sep;89(9):1206-9.

[27]. Ramchander Siwach Roop Singh Rajesh Kumar Rohilla et al Internal fixation of proximal humerus fracture by locking proximal humerus plate in elderly osteoporotic J Orthopaedic Trauma (2008) 9:149-153.

[28]. Rizwan Shahid et al, "proximal humerus fracture treated with locking compression plate" Acta Orthop. Belg., 2008, 74, 60 2-608

[29]. Kenneth A.egol, Crispin Cong, Michael Walsh et al, Early complication of proximal humerus fractures treated with locked plates, J ortho trauma 2008; 22:159-164.

[30]. Felix Brunner, Christoph Sommer, Christian Bahrs et al Open Reduction and Internal Fixation of Proximal Humerus Fractures Using a Proximal Humeral Locked Plate: A Prospective Multicenter Analysis, J Ortho Trauma 2009;23:163-172.

[31]. AA Martinez et al. "proximal humerus locking plate for proximal humerus fracture -retrospective study" journal of orthopaedic surgery2009; 17(1):10-4

[32]. MA Fazal,FS Haddad "PHILOS plate fixation for displaced proximal humeral fractures" journal of orthopaedic surgery2009;2009 17(1)15-8.

[33]. Sameer Aggarwal et al "Displaced proximal humeral fractures: an Indian experience with locking plate". Journal of Orthopaedic surgery and research2010,5:60.

[34]. Georg Osterhoff,Christian Ossendorf et al. "The calcar screw in angular stable plate fixation of proximal humerus fractures-a case study". Journal of orthopedic surgery and research 2011,6:50.

[35]. Adithya C Pawaskar, Kee-Won Lee. "locking plate for proximal humerus fracture in the elderly population:serial change in neck shaft angle" clinic ortho surg.2012 september:4(3)209-215.

[36]. Gray's Anatomy, 40th Edition, Susan Standring.

[37]. Esser, 1994. Esser RD: Open reduction and internal fixation of three- and four- part fractures of the proximal humerus. Clin Orthop Relat Res 994;299:244.

[38]. Hawkins RJ, Kiefer Gin: Internal fixation for proximal humeral Fractures Clin. Orthop-1987:223:77-85

[39]. Lorenzo FT. Osteosynthesis with Blount staples in fracture of the proximal end of the humerus: a preliminary report. J Bone Joint Surg Am 1955;37:45 - 48 .

[40]. Lind T, Kronerk, Jensen J. The epidemiology of fractures of proximal humerus. Arch Orthop Trauma Surg, 1989; 108: 285.

[41]. Kocher T. Beitrage zur Kenntnis einiger praktisch wichtige Frakturenformen. Basel: Karl Sallman Verlag; 1896. 
[42]. Marsh JL, Slongo TF, Agel J, Broderick JS, Creevey W, DeCoster TA et al. Fracture and Dislocation Classification Compendium 2007: Orthopaedic Trauma Association Classification, Database and Outcomes Committee. J Orthop Trauma 2007; 21:S1-133.

[43]. .Palvanen M, Kannus P, Parkkari J, et al: The injury mechanisms of osteoporotic upper extremity fractures among older adults: A controlled study of 287 consecutive patients and their 108 controls. Osteoporos Int 2000; 1:822-831.

[44]. Chapman JR, Henley MB, Agel J, et al. Randomized prospective study of humeral shaft fracture fixation: intramedullary nails versus plates. J Orthop Trauma. 2000; 14:162-166.

[45]. Jaberg H, Warner JJ, Jakob RP. Percutaneous stabilization of unstable fractures of the humerus. J Bone Joint Surg [Am] 1992;74A:508-15.

[46]. Movin T, Sjoden GO, Ahrengart L. Poor function after shoulder replacement in fracture patients: a retrospective evaluation of 29 patients followed for 2-12 years. Acta Orthop Scand 1998;69:392-6).

[47]. Stanley Hoppenfield Piet deBoer; Surgical exposures in Orthopaedics The anatomic approach 3rd Ed Lippincott William Wilkins.

[48]. P.Hoffmeyer: The operative management of displaced fractures of the proximal humerus JBJS (Br) 2002 May Vol 84-B No 4 Pg 469-80.

[49]. Evan L. Flatow. Fracture of the proxiaml humerus. Chapter-25, In: Text book of Rockwood and Green's fractures in adults. Vol.1, New York: Lippincott Willimas \& Wilkins, 2001: p.997-1035. 